\title{
IPS OBSERVATIONS AT MIYUN STATION, BAO
}

\author{
J. WU, X. ZHANG and Y. ZHENG \\ National Astronomical Observatories, CAS, A20 Datun Road, Beijing 100012, China; \\ E-mail:jhwu@classI.bao.ac.cn (J.Wu);zxz@class1.bao.ac.cn (X.Zhang);zyj@classI.bao.ac.cn \\ ( $Y$. Zheng)
}

\begin{abstract}
IPS observations have recently begun at Miyun Station, Beijing Astronomical Observatory. This paper briefly describes the radio telescope at Miyun Station, discusses the observation and the data reduction procedures, and presents the preliminary results of observations on IPS source $3 \mathrm{C} 48$.
\end{abstract}

\section{Introduction}

Radio waves from distant radio sources are scattered by the irregular structures of the solar wind plasma when propagating through the interplanetary space, resulting in a randomly fluctuating intensity pattern. This phenomenon is called interplanetary scintillation, or IPS. Observations of IPS give information on the solar wind speed and irregular structures in the solar wind plasma. It also provides a way of studying the sub-arcsec structure of weak radio sources, which may be inaccessible to VLBI.

IPS observations began at Miyun Station, Beijing Astronomical Observatory, in the late half of 1999. This paper describes the Miyun IPS telescope in Section 2, gives the IPS observations and data analysis procedures in Section 3 and Section 4, and presents the preliminary results of some observations on IPS source $3 \mathrm{C} 48$ in Section 5 .

\section{The Telescope}

The Meter-wave aperture Synthesis Radio Telescope (MSRT) is an array consisting of 28 paraboloidal antennae, each has a diameter of $9 \mathrm{~m}$. The antennae are aligned in East-West direction with a longest baseline of about $1.2 \mathrm{~km}$. They are divided into A and B array. The A array consists of 16 elements with separations of $72 \mathrm{~m}$ between adjacent antennae, while the B array consists of 12 elements, 6 elements located at the East of the A array and the other 6 at the West. The separation between adjacent elements is $12 \mathrm{~m}$ in the B array. The total collecting area of the MSRT is about $1600 \mathrm{~m}^{2}$, similar to the collecting area of a $47 \mathrm{~m}$ single-dish 
telescope. When the telescope is used in IPS observing mode, the effective area is about $900 \mathrm{~m}^{2}$.

The MSRT, as its name suggests, was originally designed as an aperture synthesis telescope. When it is used in IPS observations, the signals collected by the antennae are simply added in A and B array, separately. Then the A array signal and $\mathrm{B}$ array signal are correlated and recorded by computer. The operating frequency is $232 \mathrm{MHz}$.

\section{Observations}

The IPS observations began at Miyun Station, BAO at the late half of 1999. Since the collecting area of MSRT is very limited, several strong IPS sources were chosen for the initial observations, such as $3 \mathrm{C} 48$, 3C147, 3C196, 3C273, 3C279, etc.

After doing the gain and phase calibration (by pointing the telescope at Cyg A), the telescope is pointed at an IPS source and $9 \sim 20$ minutes of on-source observation is carried out. Then the telescope is moved $\sim 1.5^{\circ}$ away from the source and a $6 \sim 9$ minutes off-source observation is made. The sampling rate is $20 \mathrm{~ms}$ using a time constant of $60 \mathrm{~ms}$.

Since there is currently no delay compensation system on MSRT, the IPS observations can only be done when the source is within half an hour of the meridian.

\section{Data Analysis}

\subsection{OBTAINING THE POWER SPECTRA}

The procedures of our data analysis are similar to those used by Manoharan and Ananthakrishnan (1990) and Rao et al. (1974). First, the observed intensity fluctuations are displayed and interference is edited out. Running means over $10 \mathrm{~s}$ are subtracted from the data. Then the Fast Fourier Transform (FFT) is taken to give the power spectrum and the spectrum is smoothed over three adjacent points. These procedures are carried out on on-source data and off-source data, separately. The off-source spectrum, or the spectrum of the receiver noise, is subtracted from the on-source spectrum to give the true spectrum of the intensity fluctuations. Figure 1 shows several IPS power spectra obtained at Miyun Station, BAO.

\subsection{COMPUTING THE SCINTILLATION INDEX AND SOLAR WIND SPEED}

The degree of scintillation is defined by the scintillation index:

$$
m=\frac{\text { rms intensity fluctuation }}{\text { mean source intensity }} .
$$



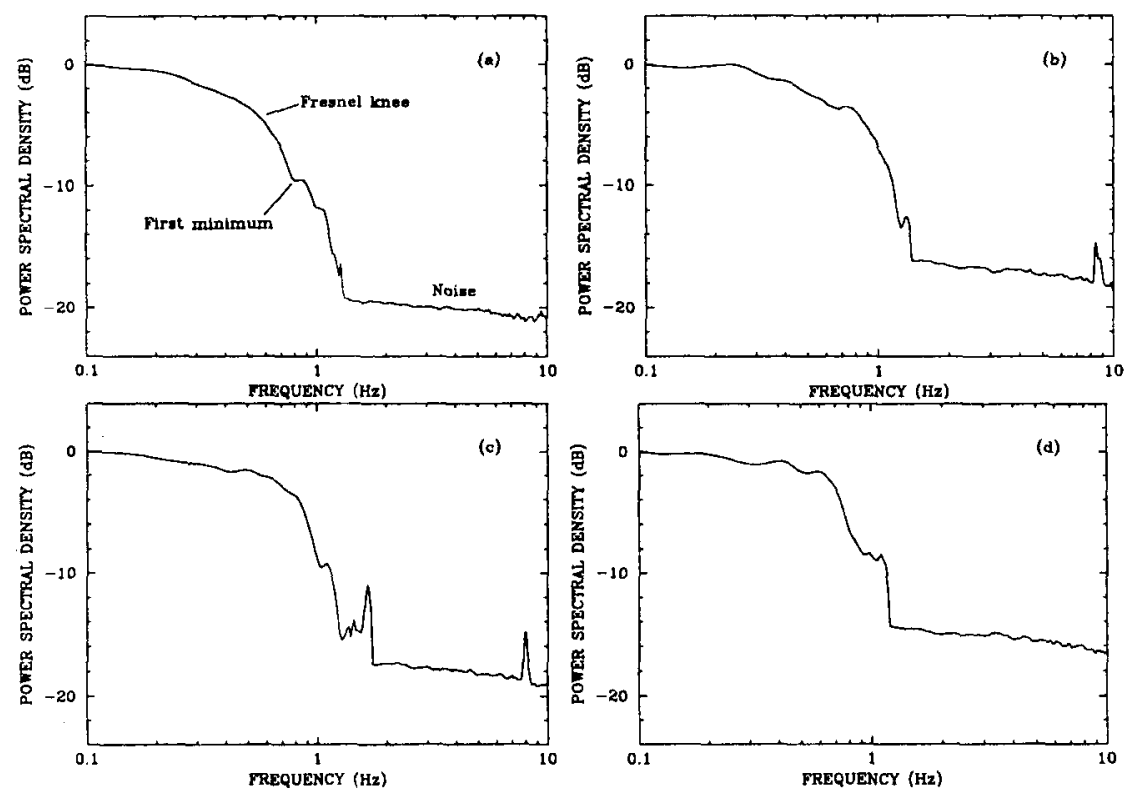

Figure I. Power spectra of IPS source 3C48 obtained at Miyun Station on 2000 April 12, May 12, 13, and 16 for (a), (b), (c), and (d) respectively.

In practice, the rms of intensity fluctuations are not a direct observable because of the receiver noise. The computational formula for scintillation index is (Oberio, 2000):

$$
m=\frac{\sqrt{\sigma_{o m}^{2}-\sigma_{o f f}^{2}}}{C_{o n}-C_{o f f}}
$$

where $C_{o n}$ and $C_{o f f}$ are the on-source and the off-source intensities respectively and $\sigma_{o n}^{2}$ and $\sigma_{o f f}^{2}$ represent the on-source and the off-source variances respectively.

There are three ways to estimate the solar wind speed (Ma, 1993).

- The speed can be estimated from the Fresnel frequency $f_{F}$ :

$$
V=f_{F} \sqrt{\pi \lambda Z} \text {. }
$$

- It can be estimated from the first minimum of the spectrum:

$$
V=f_{i} \sqrt{\lambda Z} \text {. }
$$

- It can be estimated by fitting a multi-layer 'thin phase screen' prediction to the observed spectrum.

The first two ways are straightforward but may result in large errors. The third should be more accurate than the first two, but the necessary software is still under development at Miyun Station. 


\section{Preliminary Results on $3 \mathrm{C} 48$}

Some observations were recently carried out on 3C48. Figure 1 gives the scintillation spectra obtained on four different dates. Since the collecting area of MSRT is limited, the system noise is relatively high and dominates the high-frequency $(\geq 1.3 \mathrm{~Hz})$ parts of the spectra. The noise levels are different because of the variation in phase calibration. The Fresnel knees and the first minima are clear in the low-frequency parts of the spectra. The solar wind speeds are estimated as about $423,514,554$, and $445 \mathrm{~km} \mathrm{~s}^{-1}$ for (a), (b), (c), and (d), respectively. As mentioned above, these values are only preliminary results and are not as accurate as the results that can be obtained from the model fitting which is still under development at Miyun Station.

Since the collecting area of MSRT is very limited, routine observations as in Japan and India are not possible at Miyun Station. We shall concentrate on some bright IPS sources and trace the propagation of solar wind speed variations through the interplanetary space once the delay compensation system is mounted on the MSRT.

\section{References}

Manoharan, P.K. and Ananthakrishnan, S.: 1990, Determination of solar-wind velocities using singlestation measurements of interplanetary scintillation, Mon. Not. R. Astron. Soc: 244, 691-695.

Ma, G.: 1993, PhD Thesis, Beijing Astronomical Observatory, Chinese Academy of Sciences, p. 45. Pramesh Rao, A., Bhandari, S.M. and Ananthakrishnan, S.: 1974, Observations of Interplanetary Scintillations at $327 \mathrm{MHz}$, Aust. J. Phvs. 27, 105-120.

Oberio, D.: 2000, PhD Thesis, NCRA, Tata Institute of Fundamental Research, India, p. 95. 